\title{
Elastic constants and the effect of strain on monovacancy concentration in fcc hard-sphere crystals
}

\author{
Sang Kyu Kwak and David A. Kofke \\ Department of Chemical and Biological Engineering, University at Buffalo, The State University of New York, \\ Buffalo, New York 14260-4200, USA \\ (Received 22 July 2004; published 29 December 2004)
}

\begin{abstract}
We investigate the free energy and the concentration of monovacancies in strained face-centered-cubic (fcc) hard-sphere crystals for several densities at and above melting. We use the conventional molecular dynamics method for simulations and employ a bias insertion method to extract properties of a monovacancy. We study two distinct constant-volume strains, considering a simple shear and an orthogonal expansion and contraction. Strains are examined across the linear elastic region and include also some nonlinear elastic deformations. Second-order elastic constants are reported as a function of density. The concentration of monovacancies decreases as density increases for both strained and unstrained crystals. The effect of strain is to cause the monovacancy concentration to increase by up to $72 \%$ for the expansion-contraction strain at the largest deformation studied. The effect of the shear strain is considerably less, and produces an increase in monovacancy concentration of at most $9 \%$ for the conditions studied here.
\end{abstract}

DOI: 10.1103/PhysRevB.70.214113 PACS number(s): 61.72.Ji, 62.20.Dc, 02.70.Ns, 82.70.Dd

\section{INTRODUCTION}

Vacancies are defects in crystal lattices in which atoms are absent from their expected positions. They exist in low concentration in equilibrium crystals, yet they are emphasized in studies of solid state materials because of their disproportionate effect on material properties. ${ }^{1-5}$ Known examples can be found in phenomena of photonic materials, stress relaxation, influences on the first-order transition of solid materials (with some exceptions ${ }^{6}$ ), and so on. Vacancies are able to increase the electrical conductivity by introducing discrete states in the band gap so that charge carriers (electrons or holes) can easily jump between conduction and valence bands. Local stress often resides in crystal structures due to abnormal atomistic forces around a vacancy. ${ }^{7}$ From the thermodynamic point of view, contributions of vacancies must be included for accurate free energy calculations of solid state materials.

Molecular modeling studies have provided a great deal of insight on vacancies in crystals. Bennett and Alder ${ }^{8}$ reported the equilibrium concentration of monovacancies and higher order vacancies in hard-sphere crystals by a molecular dynamics (MD) approach. The equation of state approach was taken by Speedy and Reiss ${ }^{9}$ and Bowles and Speedy. ${ }^{10}$ They reported number, size, and surface area of cavities in $D$-dimensional hard-sphere fluids and crystals. Swope and Anderson ${ }^{11}$ explored computer simulation methodologies for considering vacancies with appropriate ensembles taking into account thermodynamic constraints involved in fixing the number of lattice sites. Recently, Pronk and Frenkel ${ }^{12}$ reviewed the statistical mechanics of a crystal with point defects. They considered two approaches, using grandcanonical and isothermal-isobaric formalisms, respectively. With Monte Carlo (MC) simulation, they estimated the concentration of interstitials in hard-sphere crystals. They introduced the concept of the number of lattice sites into the grand-canonical ensemble and considered the fluctuation of the lattice sites for the equilibrium of the system.

The coupling between point defects and mechanical strain is a topic of some interest, relevant, for example, to the study of damage mechanics. However, the effects of external strain on equilibrium properties of monovacancies in a crystal system are not well studied from a molecular thermodynamic point of view (i.e., emphasizing free energies and defect equilibria). In the present study we address this issue, applying molecular simulation to study vacancies in equilibrated systems under distinct strains. We consider only monovacancies and examine them in the fcc hard-sphere crystal system. The hard-sphere model is a very well studied system, and derives its importance from its ability to describe qualitatively the behavior of atomistic crystals, and quantitatively the behavior of some colloidal materials. We use the conventional collision-based MD method ${ }^{13}$ in conjunction with the insertion-deletion bias method introduced by Pronk and Frenkel. ${ }^{12}$ In Sec. II, we briefly explain the theoretical approach of the grand-canonical scheme and ways it can be extended to examine vacancies in strained systems. In Sec. III, we describe computational details and in Sec. IV we present and discuss results. We summarize and conclude in Sec. V.

\section{THEORETICAL APPROACH}

\section{A. Free energy of monovacancy formation}

We adopt the Pronk and Frenkel ${ }^{12}$ approach to obtain the concentration of monovacancies in an otherwise perfect crystal. They present a formalism using the grand-canonical ensemble. Beginning with a system having a fixed number of lattice sites $M$, they define the change in the free energy for the creation of a single vacancy: $-f_{1}(M)=F^{(1)}-F^{(0)}$, where $F^{(n)}$ is the Helmholtz free energy of a system having $n$ vacancies. The number of lattice sites is allowed to fluctuate to adjust to the number of vacancies, and the true grand- 
canonical partition function is obtained as a sum over all states with different numbers of lattice sites,

$$
\begin{aligned}
\Xi= & \sum_{\Delta M=-\infty}^{\infty} \Xi_{M+\Delta M}^{\prime}=\sum_{\Delta M=-\infty}^{\infty} Q_{M+\Delta M} \exp [(M+\Delta M) \beta \mu] \\
& \times \exp \left[(M+\Delta M) \exp \left(-\beta\left[\mu-f_{1}(M+\Delta M)\right]\right)\right],
\end{aligned}
$$

where $\beta=1 / k_{B} T=1, k_{B}$ is Boltzmann's constant, $T$ is the temperature, $\mu$ is the chemical potential for the perfect crystal, $\mu=(F+p V) / M$ with $p$ the pressure and $V$ the volume. $Q_{M+\Delta M}$ is the canonical partition function with the lattice fluctuation $\Delta M$. Assuming that the ensemble is dominated by the largest term in the $\Delta M$ sum when in the thermodynamic limit, the maximum distribution of the ensemble is found by maximizing the exponent, which undergoes Talyor series expansion in terms of $M$. Also, it is assumed that monovacancies are noninteracting with each other due to their low concentration in the system. To this end, the maximum value of the fractional change of the number of lattice sites $\Delta M / M$ was found and thus the maximum grand canonical ensemble containing first and second orders of the leading quantity $\exp \left[-\beta\left(\mu-f_{1}(M)\right)\right]$ was obtained. The concentration of the vacancies is, then, found by using a thermodynamic relation. ${ }^{2}$ The monovacancy concentration $x_{M V}$ is thereby expressed

$$
x_{M V}=\frac{\langle n\rangle}{M}=\exp \left[-\beta\left(\mu-f_{1}\right)\right] .
$$

Since the equilibrium concentration of vacancies is generally very low, the second order term can be omitted. The argument of the exponent in Eq. (2) without a negative sign is a driving force for the insertion of a particle into a vacant site, and it is balanced by the entropic gain made in maintaining the site vacant.

\section{B. Strain energy and stress/free-energy derivative relation}

Both $\mu$ and $f_{1}$ of Eq. (2) are altered by the presence of strain, and the effect of strain on the monovacancy concentration is made through its effect on these quantities. The free energy of the strained system is defined by Wallace, ${ }^{14}$

$$
\rho f\left(\eta_{i j}\right)=\rho f(0)+T_{i j} \eta_{i j}+\frac{1}{2} C_{i j k l} \eta_{i j} \eta_{k l}+\cdots,
$$

where $1<i, j, k, l<3, \rho$ is the lattice-site number density $M / V, f(0)=F(0) / M$ is the free energy (per site) of the unstrained system, $T_{i j}$ is the stress tensor, and $C_{i j k l}$ are the second-order elastic constants, known as Young's moduli. The Einstein summation convention is assumed. Note that Eq. (3) is a series expansion of the free energy with respect to the Lagrangian strain tensor $\eta_{i j}$, which explicitly describes the state of the deformation. This tensor is defined in terms of the displacement gradients $\partial u_{i} / \partial x_{j}$,

$$
\eta_{i j}=\frac{1}{2}\left[\frac{\partial u_{i}}{\partial x_{j}}+\frac{\partial u_{j}}{\partial x_{i}}+\frac{\partial u_{k}}{\partial x_{i}} \frac{\partial u_{k}}{\partial x_{j}}\right],
$$

where $u_{i}$ are differences of positions of atoms between strained and unstrained systems, and $x_{i}$ are the atom coordinates. Crystal symmetries introduce simplifications that greatly reduce the number of distinct elastic-constant coefficients, and the Voigt notation ${ }^{15}$ is used to wrap two indices to one,$\quad(11 \rightarrow 1 ; 22 \rightarrow 2 ; 33 \rightarrow 3 ; 12,21 \rightarrow 4 ; 23,32 \rightarrow 5 ; 13,31$ $\rightarrow 6$ ). To this end, total number of second-order elastic constants of fcc crystal is reduced to three, $C_{11}, C_{12}$, and $C_{44}$.

By definition, in the linear elastic regime these elastic constants are sufficient to determine, via Eq. (3), the free energy of a strained system relative to an unstrained one. Inasmuch as this study is not restricted to the linear elastic region and moreover is confined to a constant volume, we develop a direct approach to evaluate the strain energy in cooperation with the stress/free-energy derivative relation, as given by Wallace, ${ }^{14}$

$$
\frac{\partial(\rho f)}{\partial \varepsilon}=\frac{\partial(\rho f)}{\partial \eta_{k l}} \frac{\partial \eta_{k l}}{\partial \varepsilon}=T_{i j}(\{\eta\}) \gamma_{i k} \gamma_{j l} \frac{\partial \eta_{k l}}{\partial \varepsilon},
$$

where $T_{i j}(\{\eta\})$ is a stress-tensor element in a deformed system, $\gamma_{i k}$ is an element of the inverse of the transformation tensor (detailed description can be found from Ref. 14), and $\varepsilon$ is the strain parameter. We evaluate free energies in the strained system by numerical integration of Eq. (5), using simulation data for $T_{i j}(\{\eta\})$. We do this, rather than use Eq. (3), to avoid errors resulting from nonlinear elastic behavior [i.e., neglect of high order terms in Eq. (3)].

\section{Strain effects on monovacancy concentration}

Our aim in the present work is to determine the change in the equilibrium monovacancy concentration as different strains are applied to the crystal. A closely related problem is to determine the local effect of monovacancies on the elastic constants. A straightforward way to approach this calculation is to adopt the formalism of Pronk and Frenkel, ${ }^{12}$ modified only in that it is applied to a strained system. We call this method the insertion-deletion simulation (IDS) approach. Thus the monovacancy concentration is again given by Eq. (2), but with the chemical potential $\mu$ and the free energy of monovacancy formation $f_{1}$ both taken for the strained crystal. The ratio of the monovacancy concentration in the strained to the unstrained crystal is then

$$
\frac{x_{M V}(\eta)}{x_{M V}(0)}=\exp \left[-\beta\left(\left(\mu(\eta)-f_{1}(\eta)\right)-\left(\mu(0)-f_{1}(0)\right)\right)\right],
$$

where $(\eta)$ indicates the strained system, and (0) the unstrained one.

Alternatively, we can examine the change in the free energy upon the application of the strain to a perfect crystal with and without a monovacancy. We call this method the strain energy calculation (SEC) approach. A key quantity in Eq. (6) is the difference in $f_{1}$ between the strained and unstrained systems. In the IDS approach this difference is evaluated by performing insertion/deletion averages in the strained and unstrained systems, respectively, to get each $f_{1}$, and taking their difference. Thus 


$$
\begin{aligned}
f_{1}(\eta)-f_{1}(0) & =\left(F^{(0)}(\eta)-F^{(1)}(\eta)\right)-\left(F^{(0)}(0)-F^{(1)}(0)\right) \\
& =\left(F^{(0)}(\eta)-F^{(0)}(0)\right)-\left(F^{(1)}(\eta)-F^{(1)}(0)\right),
\end{aligned}
$$

where $F$ is the total free energy of the system. The latter equality indicates evaluation of the difference in $f_{1}$ by measuring the free-energy change associated with strains in crystals with 0 and 1 vacancies, respectively. Also, to the extent that the system obeys linear elastic behavior, the difference in $f_{1}$ relates to the change in the stress tensor and elastic constants with the addition of a vacancy,

$$
f_{1}(\eta)-f_{1}(0)=V\left[\left(T_{i j}^{(0)}-T_{i j}^{(1)}\right) \eta_{i j}+\frac{1}{2}\left(C_{i j k l}^{(0)}-C_{i j k l}^{(1)}\right) \eta_{i j} \eta_{k l}\right] .
$$

\section{SIMULATION DETAILS}

We conducted MD simulations of systems of hard spheres (diameter $\sigma=1)$ occupying an $M=108$-site fcc lattice $(3 \times 3$ $\times 3$ unit cells), examining volumes $V$ from melting $\left(1.359 V_{\mathrm{o}}\right)$ to $1.2 V_{\mathrm{o}}$, where $V_{\mathrm{o}}=M \sigma^{3} / \sqrt{2}$ is the volume of the system at close packing. Finite-size effects were examined by performing some simulations of systems with 256 lattice sites $(4 \times 4 \times 4$ unit cells) and differences were found to be negligible over densities of interest. Keeping the volume constant, the symmetry of the fcc crystal provides for only two independent deformations. We chose one as a shear strain in the $x-y$ plane, with a strain parameter $\varepsilon$ defined to produce the coordinate transformation,

$$
x^{\prime}=x, \quad y^{\prime}=y+\varepsilon x, \quad z^{\prime}=z,
$$

where $x^{\prime}, y^{\prime}, z^{\prime}$ are coordinates of the strained system, $x, y$, $z$ are those for the unstrained system. The other strain expands uniformly along the $x$ axis and contracts along the $y$ axis (the expansion-contraction strain) defined by

$$
x^{\prime}=(1+\varepsilon) x, \quad y^{\prime}=\left(\frac{1}{1+\varepsilon}\right) y, \quad z^{\prime}=z
$$

Figure 1 illustrates these strains. Note that both deformations keep the volume of the system constant. Within the assumption of linear elasticity, the concentration and the formation free-energy of monovacancies for any combination of the above strains, plus those that do change the volume, can be extracted from analysis of those two deformations with knowledge of the dependence of the vacancy properties on density for the unstrained system.

The stress tensor is obtained by summing contributions from the collision virial over all pair collisions processed in the simulation. ${ }^{13}$ Then the free energy as a function of strain is obtained by numerical integration of the stress-tensor data according to Eq. (5). For the shear strain [Eq. (9)], this equation reduces to

$$
\frac{\partial(\rho f)}{\partial \varepsilon}=T_{12}(\varepsilon)-2 T_{12}(\varepsilon) \varepsilon^{2}+T_{22}(\varepsilon) \varepsilon^{3}
$$

and for the expansion-contraction strain [Eq. (10)] it is

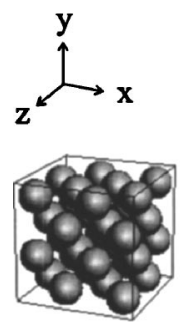

(a)

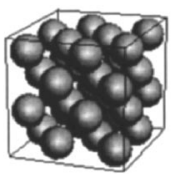

(b)

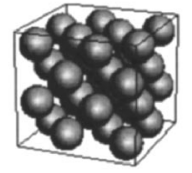

(d)

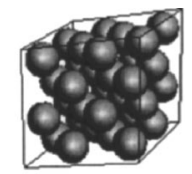

(c)

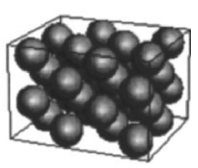

(e)
FIG. 1. Illustration of strains defined by Eqs. (9) and (10), using 32 particles of the fcc system $(2 \times 2 \times 2$ unit cells). (a) The unstrained system; (b) shear-strained system with $\varepsilon=0.03$ and (c) with $\varepsilon=0.2$; (d) expansion-contraction strained system with $\varepsilon=0.03$ and (e) with $\varepsilon=0.2$. High-strain $(\varepsilon=0.2)$ images are included only to illustrate better the nature of the strains, as the distortions are barely visible for $\varepsilon=0.03$.

$$
\frac{\partial(\rho f)}{\partial \varepsilon}=\frac{T_{11}(\varepsilon)-T_{22}(\varepsilon)}{1+\varepsilon} .
$$

The integration was accomplished by fitting each right-hand side to a polynomial quadratic in $\varepsilon$, which describes the data very well over the conditions studied.

Elastic constants were obtained by differentiating Eq. (3) with respect to $\varepsilon$ for each strain and equating to Eqs. (11) and (12) and in the limit $\varepsilon \rightarrow 0$. $C_{44}$ can be directly evaluated from the shear-strain data, via

$$
\frac{\partial(\rho f)}{\partial \varepsilon}=\left(C_{44}-p\right) \varepsilon .
$$

The expansion-contraction data yield information regarding the combination of $C_{11}$ and $C_{12}$,

$$
\frac{\partial(\rho f)}{\partial \varepsilon}=2\left(C_{11}-C_{12}-2 p\right) \varepsilon,
$$

where in both equations the term $T_{11}(0)$ is replaced by the negative of the pressure, $-p$, as the equations apply for the limit of an unstrained system. Decoupling of $C_{11}$ and $C_{12}$ is completed through their connection to the isothermal compressibility, which satisfies ${ }^{14,15}$

$$
\rho\left(\frac{\partial p}{\partial \rho}\right)_{T}=\frac{1}{3}\left(C_{11}+2 C_{12}+p\right) .
$$

We examined strain parameters $\varepsilon=0.0025,0.005,0.0075$, $0.01,0.02$, and 0.03 . To calculate elastic constants, we could use the linear term of a quadratic fit of $\partial(\rho f) / \partial \varepsilon$ for the left-hand side of Eqs. (13) and (14). We examined instead using a linear fit of the data only up to $\varepsilon=0.0075$ for the shear strain and 0.005 for the expansion-contraction strain, and found little difference in the results; the data we report are based on this latter approach. We used the equation of state of Speedy ${ }^{16}$ to determine $(\partial p / \partial \rho)_{T}$ for the unstrained system at each density for use in Eq. (15). 


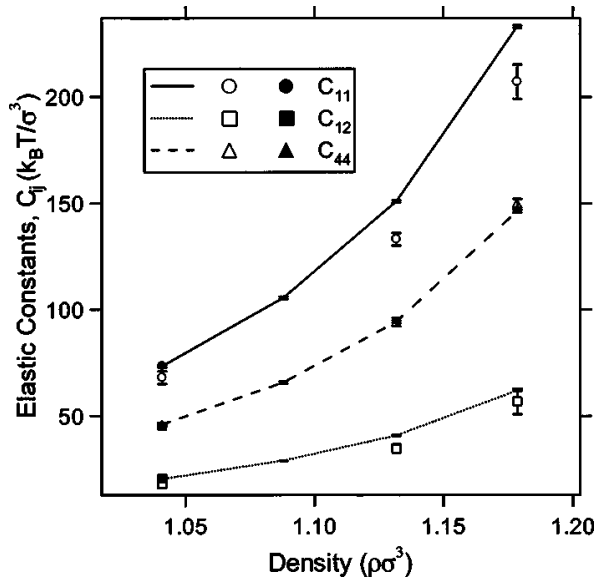

FIG. 2. Density dependence of elastic constants. Solid, dashed, and dotted lines are simulation results; error bars indicate location of data points, which are joined by the lines as a guide to the eye. Open markers are elastic constants of Frenkel and Ladd (Ref. 17). Filled markers are elastic constants of Runge and Chester (Ref. 15).

We evaluated the free energy of monovacancy formation $f_{1}$ using the bias particle-insertion method as given by Pronk and Frenkel ${ }^{11}$ which is applicable also to strained systems. The quantity $f_{1}$ is given by

$$
f_{1}=-k_{B} T \ln \left(\frac{v P_{\mathrm{acc}}(v)}{P_{\mathrm{rem}}(v) \Lambda^{3}}\right),
$$

where $P_{\text {acc }}(v)$ is the fraction of time in which no overlap with another particle is encountered upon random insertion of a particle into a bias subcell of volume $v$, and $P_{\text {rem }}(v)$ is the fraction of the time that a simulated particle is observed to lie inside its assigned subcell. Note that these simulations are microcanonical simulations so that the number of particles in the system does not change; only trial insertions/ deletions are examined. The de Broglie wavelength term $\Lambda^{3}$ cancels with the ideal part of the chemical potential when combined in Eq. (2). The natural choice of the insertion region is the Wigner-Seitz (WS) cell for the lattice site associated with the vacancy. However, most of the WS cell region is not accessible to a sphere center so, following Pronk and Frenkel ${ }_{1}^{11}$ a subcell was introduced to increase the efficiency of successful insertion trials. For the subcell we chose (for convenience) a cubic box, no larger than a size that inscribes the WS cell, so that the subcells do not overlap each other in space. For the unstrained system, the largest such subcell was used; examination of smaller cell sizes found no effect on the results for $f_{1}$. Full-size inscribed cells would overlap in the strained systems, so for those simulations we employed subcells of volume $73 \%$ of the unstrained inscribable size. Note that care must be taken to avoid spurious results arising from center-of-mass drift of the system, especially at the melting density. For the final results, approximately $1.0-5.0 \times 10^{5}$ collisions per particle for the insertion and the deletion simulations were done after an equilibration period of $5 \times 10^{4}$ collisions per particle.

\section{RESULTS AND DISCUSSION}

Figure 2 presents the results of simulations performed to verify calculation of the elastic constants and the simulation approach; our data are presented also in Table I, along with the inverse isothermal compressibility from Speedy's equation of state. ${ }^{16}$ At melting we have good agreement with the results of Runge and Chester, ${ }^{15}$ which are available only at this density. Runge and Chester employed MC simulation and applied the method of variation of density to evaluate $(\partial p / \partial \rho)_{T}$. On the other hand, our results for $C_{11}$ and $C_{12}$ differ from the data reported by Frenkel and Ladd ${ }^{17}$ over the region of density studied here. The difference may lie in the manner used to decouple $C_{11}$ and $C_{12}$. In support of the validity of our data, we point out that the Frenkel and Ladd elastic constants are not consistent with the isothermal compressibility via Eq. (15) using Speedy's equation of state ${ }^{16}$ for $(\partial p / \partial \rho)_{T}$.

Figures 3 and 4 show the combination of elastic constants as a function of strain. In particular, Fig. 3 shows $C_{44}-p$ as a function of $\varepsilon$, minus its value as $\varepsilon \rightarrow 0$, while Fig. 4 presents the same for $2\left(C_{11}-C_{12}-2 p\right)$. At sufficiently high strain parameter the elastic constants start to deviate from the zerostrain limiting values, indicating departure from the linear

TABLE I. Monovacancy concentration, elastic constants, and the inverse isothermal compressibility [according to Speedy's equation of state (Ref. 16)] for the unstrained crystal. Values are given as a function of volume $V$ relative to the close-packed volume $V_{\mathrm{o}}$ (largest value corresponds to melting). Confidence limits (67\%) for last digit of each quantity are indicated by values in parentheses. Elastic constants and the inverse isothermal compressibility are given in units of $k_{B} T / \sigma^{3}$.

\begin{tabular}{|c|c|c|c|c|c|c|c|}
\hline \multirow[b]{2}{*}{$V / V_{\mathrm{o}}$} & \multicolumn{3}{|c|}{$x_{M V}$} & \multirow[b]{2}{*}{$C_{11}$} & \multirow[b]{2}{*}{$C_{12}$} & \multirow[b]{2}{*}{$C_{44}$} & \multirow[b]{2}{*}{$\rho(\partial p / \partial \rho)_{T}$} \\
\hline & This work & $\begin{array}{c}\text { Bennett and } \\
\text { Alder }^{\mathrm{a}}\end{array}$ & $\begin{array}{c}\text { Bowles and } \\
\text { Speedy }^{\mathrm{b}}\end{array}$ & & & & \\
\hline 1.359 & $2.24(1) \times 10^{-4}$ & $2.3(5) \times 10^{-4}$ & $2.3(4) \times 10^{-4}$ & $73.4(5)$ & $20.5(2)$ & $46.1(5)$ & 42.03 \\
\hline 1.3 & $3.41(2) \times 10^{-5}$ & $4.0(4) \times 10^{-5}$ & $3.1(5) \times 10^{-5}$ & $105.5(6)$ & $29.1(2)$ & $65.7(6)$ & 59.19 \\
\hline 1.25 & $3.98(2) \times 10^{-6}$ & $4.3(4) \times 10^{-6}$ & $4.1(6) \times 10^{-6}$ & $150.8(6)$ & $40.9(2)$ & $94.2(6)$ & 83.10 \\
\hline 1.2 & $1.774(6) \times 10^{-7}$ & & $1.8(2) \times 10^{-7}$ & $232.6(7)$ & $61.9(3)$ & $146.3(7)$ & 125.75 \\
\hline
\end{tabular}

Reference 8 .

${ }^{\mathrm{b}}$ Reference 10 . 


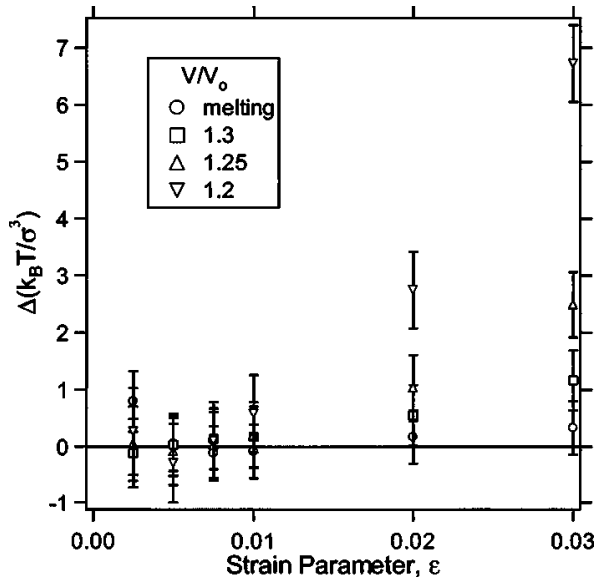

FIG. 3. The quantity $\Delta$, which is defined here as $C_{44}-p$ as a function of the strain $\varepsilon$ minus its limiting value for $\varepsilon \rightarrow 0$. Values of $V / V_{\mathrm{o}}$ indicate the system volume relative to the close-packed volume.

elastic region. For the case of the shear strain, Fig. 3, at melting linear behavior is observed over the entire range of strains studied, while at the highest density nonlinear behavior is observed at about $\epsilon=0.01$. The linear regime is much more restricted for the expansion-contraction strain, Fig. 4, where for all densities the linear regime extends no further than $\varepsilon=0.0075$. A perfect fcc lattice can be sheared up to strain parameter of about 0.21 at the melting density without overlap of spheres located at each site; for the expansioncontraction strain the corresponding maximum is 0.26 .

Now we turn to results for the free energy of formation of a monovacancy in the unstrained system. The values of $f_{1}$ and the chemical potential $\mu$ are shown in Fig. 5 over the range of density of interest. Values of $f_{1}$ increase slightly as density increases, which means that the entropy gain of neighboring atoms from the vacancy formation is greater at higher density. Offsetting this is a much greater increase in the chemical potential with density, leading to a net increase in $\mu-f_{1}$, the driving force to fill the vacancy. Consequently

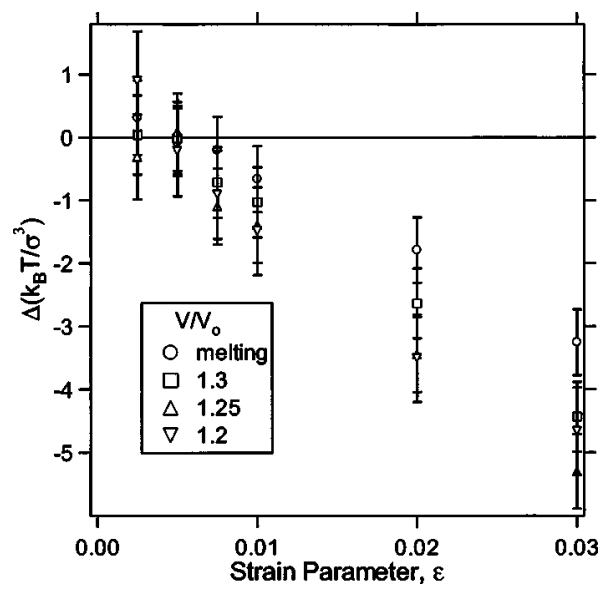

FIG. 4. The quantity $\Delta$, which is defined here as $2\left(C_{11}-C_{12}\right.$ $-2 p)$ as a function of the strain $\varepsilon$ minus its limiting value for $\varepsilon$ $\rightarrow 0$. Values of $V / V_{\mathrm{o}}$ indicate the system volume relative to the close-packed volume.

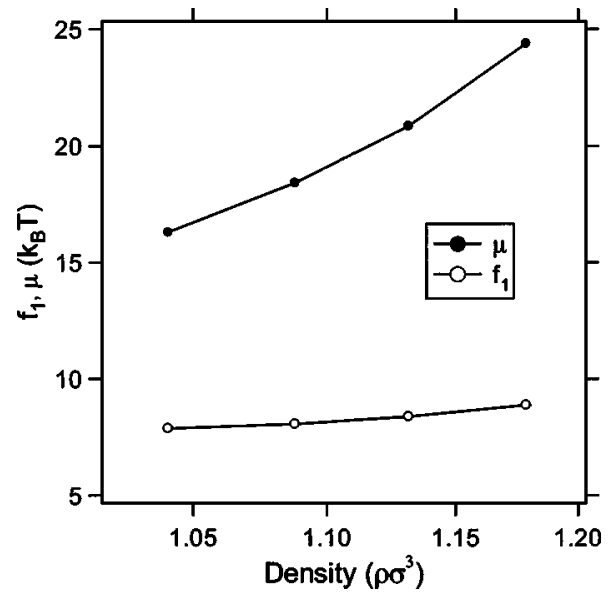

FIG. 5. Free energy of monovacancy formation $f_{1}$ and the chemical potential $\mu$ for the unstrained crystal, as a function of density. Determination of $f_{1}$ is as described in the text; $\mu$ is determined by thermodynamic integration using Speedy's equation of state (Ref. 16) from the melting point, at which $\mu$ is given by the data of Frenkel and Ladd (Ref. 18).

the concentration of monovacancy is, as expected, decreased with increasing density. Results are presented in Table I, where they are compared to the simulation data of Bennett and Alder $^{8}$ and the monovacancy-concentration model of Bowles and Speedy. ${ }^{10}$ The agreement is very good, and within the confidence limits of the calculations.

Next, we investigate strain effects on the free energy and the concentration of a monovacancy in the equilibrated system. Results are presented in Fig. 6. We find some significant effects on the monovacancy concentration depending on the nature of the strain. Generally, strain effect on a low-density solid is smaller than on one at high density. For the case of the shear strain the monovacancy concentration increases by $4 \%$ to $8 \%$, depending on density, at the maximum strain

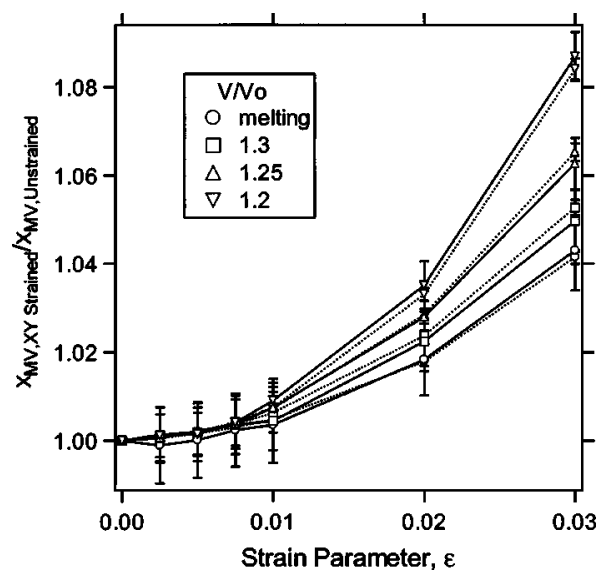

FIG. 6. Normalized monovacancy concentration as a function of strain for a shear deformation. $x_{M V \text {,Unstrained }}$ is the monovacancy concentration of the unstrained system and $x_{M V, X Y S t r a i n e d}$ the concentration in the shear-strained system. Solid lines represent results from the IDS approach and dotted lines from the SEC approach. Values of $V / V_{\mathrm{o}}$ indicate the system volume relative to the closepacked volume. 


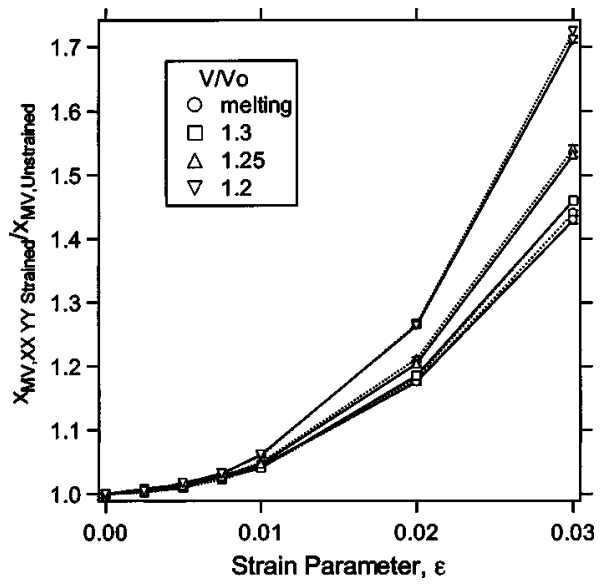

FIG. 7. Normalized monovacancy concentration as a function of strain for an expansion-contraction deformation. $x_{M V \text {,Unstrained }}$ is the monovacancy concentration of the unstrained system and $x_{M V, X X Y Y, \text { Strained }}$ the concentration in the expansion-contraction strained system. Solid lines represent results from the IDS approach and dotted lines from the SEC approach. Values of $V / V_{\mathrm{o}}$ indicate the system volume relative to the close-packed volume.

studied here. Qualitatively similar behavior occurs for the expansion-contraction case, as shown in Fig. 7. The effect however is much larger, with the concentration increasing at largest strain by $42 \%$ to $72 \%$. Considering the restriction of constant volume, this is a quite interesting result since no extra vacant volume is introduced to the original vacant site. For both types of strain, the effect on $\mu$ [in Eq. (6)] is observed to be small (not shown), and the change in the vacancy concentration is due largely to the effect of strain on $f_{1}$. This phenomenon can be explained in terms of a cell model, which considers the volume accessible to a sphere in its Wigner-Seitz cell in a distorted lattice with all other atoms constrained to their lattice sites. Numerical studies of such a system show that the shear strain causes a smaller decrease in accessible volume in comparison to a corresponding expansion-contraction strain. This effect shows up in $f_{1}$, causing it to increase toward $\mu$ and thereby decreasing the driving force to fill the vacancy. As the magnitude of the strain becomes larger, this effect grows accordingly.

Finally, we note that the results of SEC method and the IDS method are, within their confidence limits, in perfect agreement for all conditions and strains studied.

\section{CONCLUSION}

In summary, we performed MD simulations to evaluate the elastic constants and the monovacancy concentration of a system of fcc hard spheres. Both properties were measured as a function of strain, considering two types of constantvolume deformation: shear and expansion-contraction. As expected, the equilibrium concentration of monovacancy decreases as density increases, and this behavior holds also for the strained systems. Investigation of strain effects on monovacancy finds that strain increases the monovacancy concentration, and that this effect is enhanced with increasing density. The expansion-contraction strain shows a greater influence on the properties of monovacancy than the shear strain.

\section{ACKNOWLEDGMENTS}

This work is supported by the Division of Chemical Sciences, Office of Basic Energy Sciences of the U.S. Department of Energy (Contract No. DE-FG02-96ER14677). Computer resources have been provided by the University at Buffalo Center for Computational Research.
${ }^{1}$ M. P. Scripsick et al., J. Appl. Phys. 73, 1114 (1993).

${ }^{2}$ R. J. Gambino, R. R. Ruf, and P. Fumagalli, J. Appl. Phys. 73, 6109 (1993).

${ }^{3}$ D. T. Morelli and C. Uher, Appl. Phys. Lett. 63, 165 (1993).

${ }^{4}$ T. Mattila, S. Poykko, and R. M. Nieminen, Phys. Rev. B 56, 15665 (1997).

${ }^{5}$ P. Boguslawski, E. L. Briggs, and J. Bernholc, Phys. Rev. B 51, 17255 (1995).

${ }^{6}$ M. A. Bates and D. Frenkel, Phys. Rev. E 61, 5223 (2000).

${ }^{7}$ S. H. Jhi, S. G. Louie, M. L. Cohen, and J. Ihm, Phys. Rev. Lett. 86, 3348 (2001).

${ }^{8}$ C. H. Bennett and B. J. Alder, J. Chem. Phys. 54, 4796 (1971).

${ }^{9}$ R. J. Speedy and H. Reiss, Mol. Phys. 72, 999 (1991).
${ }^{10}$ R. K. Bowles and R. J. Speedy, Mol. Phys. 83, 113 (1994).

${ }^{11}$ W. C. Swope and H. C. Andersen, Phys. Rev. A 46, 4539 (1992).

${ }^{12}$ S. Pronk and D. Frenkel, J. Phys. Chem. B 105, 6722 (2001).

${ }^{13}$ M. P. Allen and D. J. Tildesley, Computer Simulation of Liquids (Clarendon, Oxford, 1987).

${ }^{14}$ D. C. Wallace, in Solid State Physics: Advances in Research and Applications, edited by H. Ehrenreich, F. Seitz, and D. Turnbull (Academic, New York, 1970), p. 301.

${ }^{15}$ K. J. Runge and G. V. Chester, Phys. Rev. A 36, 4852 (1987).

${ }^{16}$ R. J. Speedy, J. Phys.: Condens. Matter 10, 4387 (1998).

${ }^{17}$ D. Frenkel and A. J. C. Ladd, Phys. Rev. Lett. 59, 1169 (1987).

${ }^{18}$ D. Frenkel and A. J. C. Ladd, J. Chem. Phys. 81, 3188 (1984). 